\title{
Vibration Monitoring System for a Transmission Shaft in Real Time Using an Eddy Current Displacement Sensor ${ }^{\dagger}$
}

\author{
Toshihiko Yamaguchi*, Yoshiro IWaI*, Sigeru InagakI** and Masahiro UedA***
}

\begin{abstract}
A system for monitoring the vibration of a transmission shaft by means of an eddy current displacement sensor has been developed, which provides the Lissajous figures and the occupation distribution of a single revolution in real time. The sampling frequency of data acquisition was $5 \mathrm{kHz}$, which is sufficient for the speed of 1600 cycles/min used in many plants. The measurement error of the system is within $30 \mu \mathrm{m}$, which is precise enough for our purposes.
\end{abstract}

Key Words : monitoring system, eddy current displacement sensor, vibration, transmission shaft

\section{Introduction}

The vibration of a power transmission shaft is a direct consequence of rotation. It causes and therefore contributes to the risk of accident. Real-time monitoring of the vibration by the use of Lissajous figures and its analysis by means of spectral analysis are, obviously, highly desirable for many plants such as power plants and manufacturing plants. A non-contact method is preferred for this purpose.

Some vibration detection methods have been developed that use lasers to diagnose metal fatigue ${ }^{1}$. These methods can be used effectively both in air and in clean water, but cannot be used in contaminated water because the output signal, i.e., light intensity, of the sensor becomes noisy due to the contamination. A detector which makes use of an electromagnetic force has also been developed ${ }^{2}$. This can measure and diagnose only the threshold of bearing wear : it cannot analyze the vibration in real time, which can be obtained by means of Lissajous figures.

In this paper, we developed a practical system for monitoring the shaft vibration of a drain pump using an eddy current displacement sensor.

\section{Method and System}

The principle of the eddy current displacement sensor

' Presented at JSAP Hokuriku-Shinetsu Branch Symposium (2001 • 12)

* Department of Mechanical Engineering, Faculty of Engineering, Fukui University, Fukui

** Technical Support Team, Plant Management Headquarters, the Japan Atomic Power Company, Tokyo

*** Department of Human Ecology and Technology, Faculty of Education and Regional Studies, Fukui University, Fukui

(Received June 25, 2002)

(Revised August 5, 2002) described in this study is based on electromagnetic induction. The sensor creates a high frequency electromagnetic field in front of it. An eddy current is induced in a metal when the metal is put into the high frequency electromagnetic field, and the output of the sensor is decreased by this eddy current. The output is linearly proportional to the distance between the sensor and the metal, i.e., a transmission shaft in this case. The data acquisition rate of the sensor, in other words, the sampling frequency, is $5 \mathrm{kHz}$, which is sufficiently high for a practical motor. These two features make it possible to monitor vibration for a revolution speed of 1600 cycles/min in real time.

Fig. 1 shows the setup of the preliminary experiment carried out on the shaft of a drilling machine. A metal disc of $150 \mathrm{~mm}$ in diameter and $20 \mathrm{~mm}$ in thickness was fixed to the motor shaft. Two sensors were mounted close to the disc at a right angle to each other in order to observe the two-dimensional motion of the shaft, i.e., on both the $\mathrm{X}$ and $\mathrm{Y}$-axes. It is thus possible to generate Lissajous figures of the motion.

Fig. 2 shows a block diagram of the system. Both

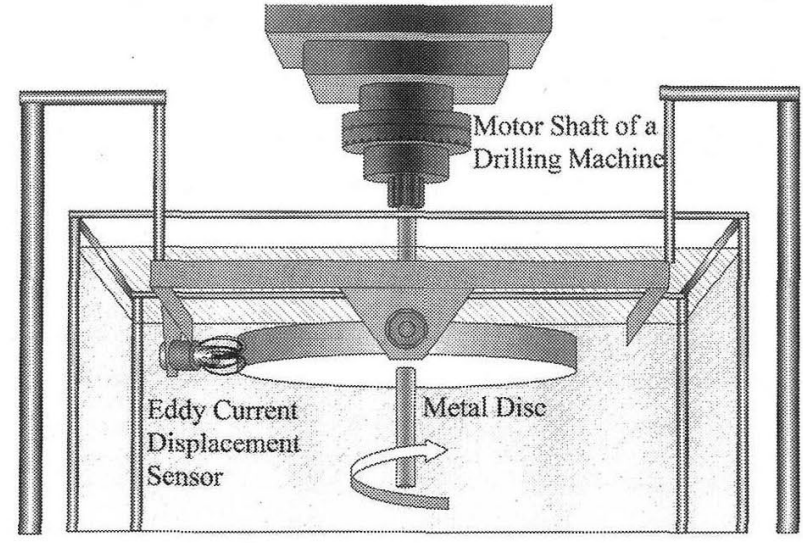

Fig. 1 Experimental setup for a preliminary experiment carried out on the shaft of a drilling machine 


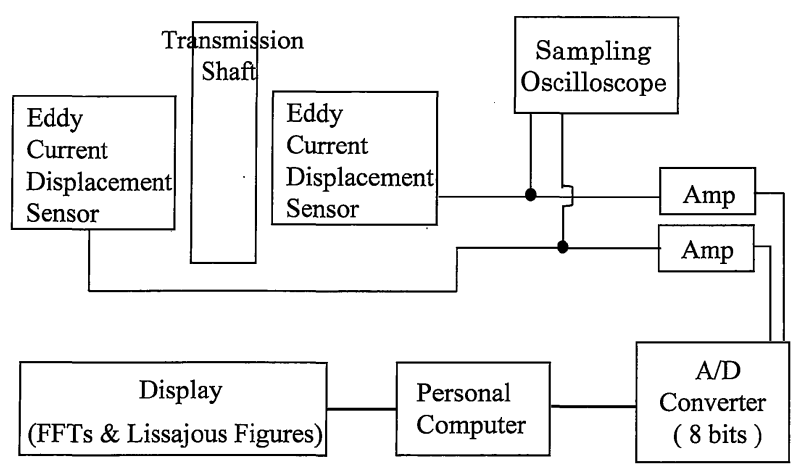

Fig. 2 Block diagram of the system

analog signals on each sensor, i.e., the outputs, were amplified to the proper level and converted to a digital signal by an $\mathrm{A} / \mathrm{D}$ converter of 8 bits with a sampling frequency of $5 \mathrm{kHz}$. The digital signal was then received on a sampling oscilloscope and personal computer in order to observe the vibration in real time, and these signals were then translated into Lissajous figures.

\section{Experimental Results}

The preliminary experiment was carried out by means of the experimental setup shown in Fig. 1. Measurements were performed with the metal in air and in water. The revolution speed was adjusted to $1600 \mathrm{cycles} / \mathrm{min}$ in air. It was, however, slightly decreased to about 1540 cycles/min in water due to the water's viscosity. The sampling frequency of measurement was $5 \mathrm{kHz}$ in all the experiments in this study. Specifically, a period of revolution, i.e., one cycle, was divided into $188(=60 \times 5000 / 1600)$ equal intervals at $1600 \mathrm{cycles} / \mathrm{min}$; in other words, one cycle was composed of 188 samples.

Fig. 3 shows Lissajous figures in a stationary revolution state, (a ) at $1600 \mathrm{cycles} / \mathrm{min}$ in air, and (b) at 1540 cycles/min in water. This expresses the deviation of the center of revolution from the stationary center, i.e., the trajectory of the center of revolution in real time. One hundred revolutions were included in these Lissajous figures. The center of the Lissajous figures, i.e., the position of the stationary center, expresses the mean distance of the sensor from the metal surface in this experiment. The experiments were carried out using other mean sensor distances. The same results were obtained with only a shift of position of the stationary center, from which the sensitivity was found to be constant. The deviations from the stationary state on both the $\mathrm{X}$ - and $\mathrm{Y}$-axes were found to be about $0.32 \mathrm{~mm}$ in peak to peak value when the disc was rotated at $1600 \mathrm{cycles} / \mathrm{min}$ in air (Fig. 3(a)). The deviation increased slightly to about $0.36 \mathrm{~mm}$ in water (Fig. 3(b)). The main reasons for an empty region
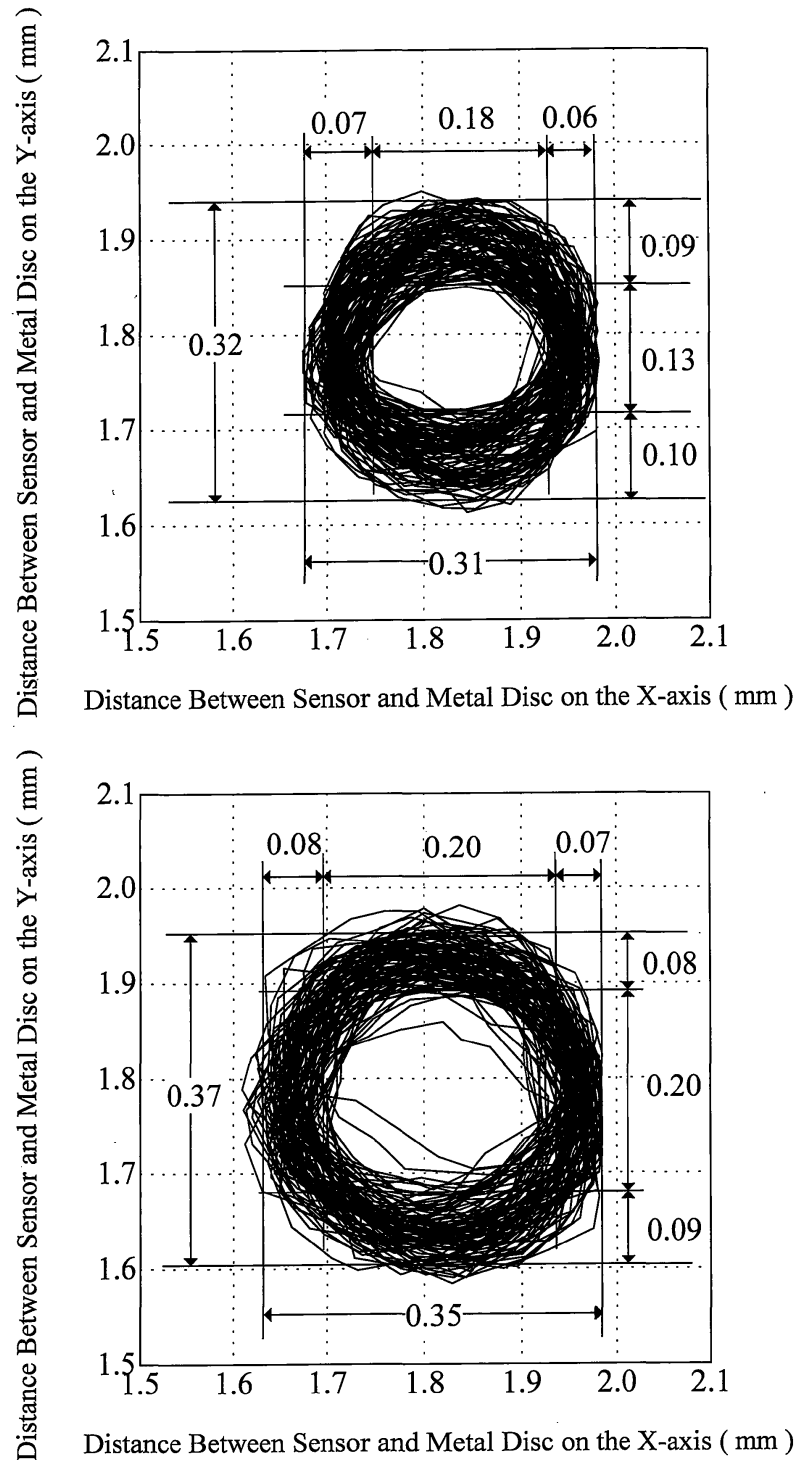

Fig. 3 Lissajous figures of the motion, i.e., the trajectory of the center revolution, of the disc. (a) $1600 \mathrm{cycles} / \mathrm{min}$ in air, and (b) $1540 \mathrm{cycles} / \mathrm{min}$ in water

around the center of revolution is due to the eccentricity of the metal disc to the revolution center caused by an error in matching the disc center to the revolution center, runout of the center due to centrifugal force, and the overload caused by the viscosity of water. The size of the filled region expresses the amplitude of the deviation.

Fig. 4 shows an occupation distribution of the center of revolution for the results given in Fig. 3(b). One dot expresses the mean value for 10 samples, and $1923(=(1 /$ 26) $\times 100 \times 5000 / 10$ ) dots are included in this figure. The sample number for the average is an important factor in analyzing a vibration within a period in real time, because the average over a period becomes a constant value, which indicates the position of the center of revolution. The two expressions shown in Figs. 3 and 4 are essentially identical 


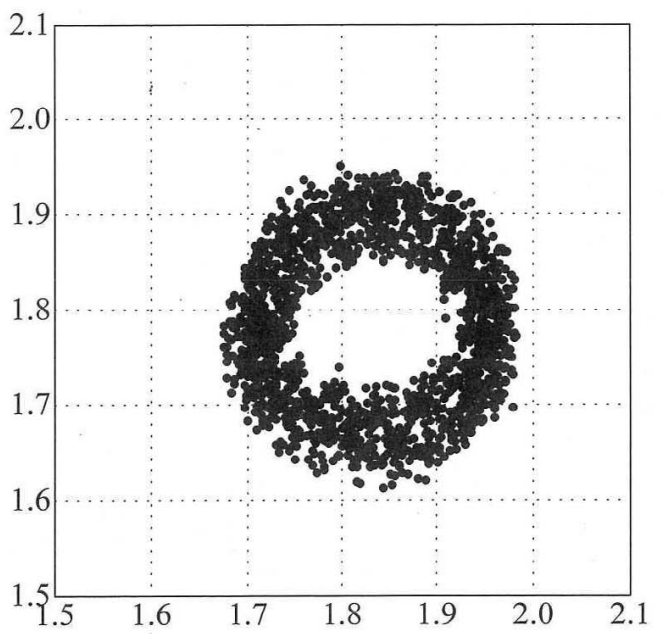

Distance Between Sensor and Metal Disc on the X-axis ( $\mathrm{mm}$ )

Fig. 4 Occupation distribution of the center of revolution for the results given in Fig. 3 (b)
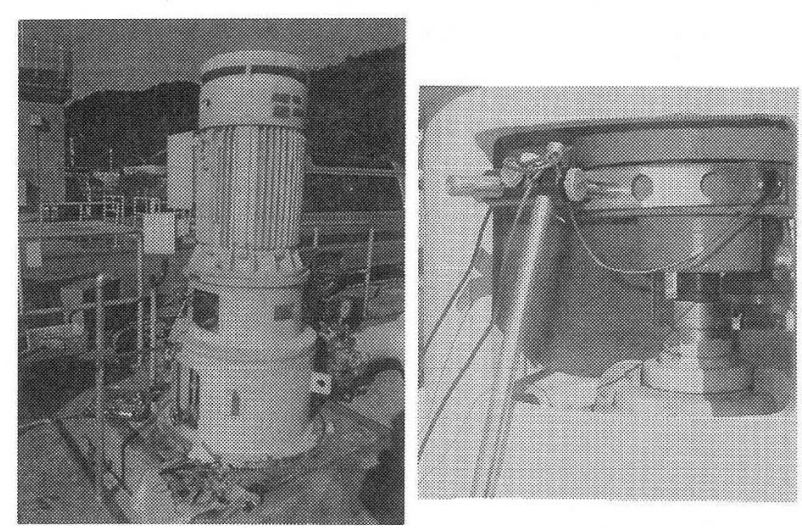

Fig. 5 Experimental setup for the plant under consideration

because Fig. 3 is merely the combination of the dots shown in Fig. 4. Thus, Fig. 3 illustrates the trajectory itself from the onset of the revolution, and Fig. 4 shows the occupation distribution of the center of revolution during the stationary state.

Fig. 5 shows the experimental setup for the plant under consideration. The experiment was carried out using a drain pump at the Tsuruga Power Station of the Japan Atomic Power Company.

Fig. 6 shows the occupation distribution for this specific drain pump from the onset of the revolution $(t=0)$ to one hundred periods $(t=100 \mathrm{~T})$. The amplitudes of deviation were found to be about $0.3 \mathrm{~mm}$ in peak to peak value on both the $\mathrm{X}$ - and $\mathrm{Y}$-axes, and to be almost the same in the stationary state. The empty region around the center of revolution was not found in this specific drain pump, indicating that the pump had little eccentricity in any direction, and further a run-out of the center of the shaft due to centrifugal force was restricted considerably by means of

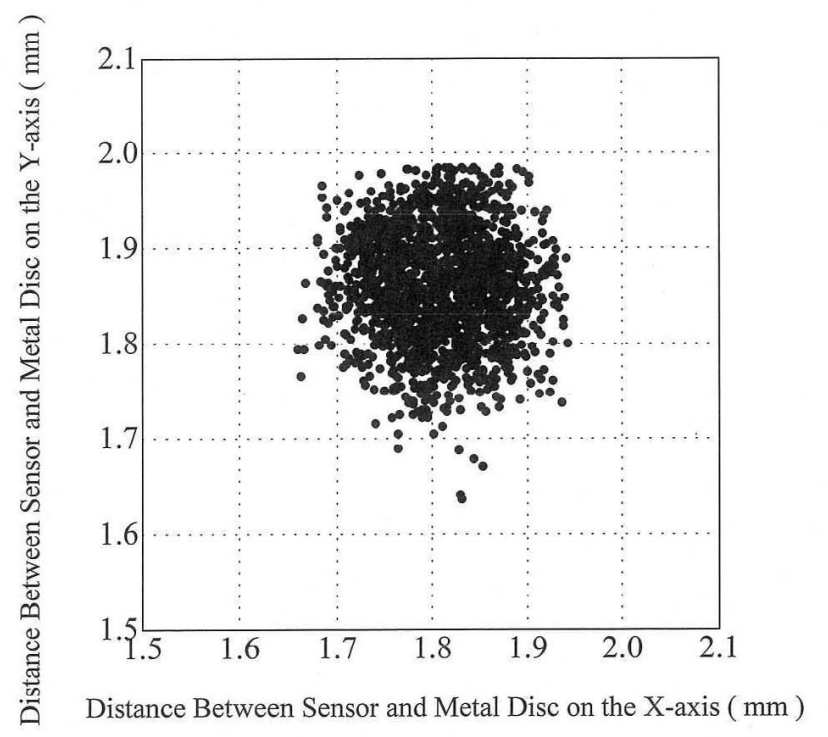

Fig. 6 Occupation distribution of the center of revolution for the drain pump in air

\section{a bearing.}

The real-time monitoring system discussed in this study is useful for analyzing bearing wear. The wear increases at the dense region of the trajectory, i.e., at a high occupation distribution, since the disc deviates to that region more frequently. Furthermore, the enlargement of the trajectory region expresses the wear of the bearing stand, since the trajectory will certainly cover all movable regions over a long period of time.

\section{Conclusion}

A real-time method has been developed and applied in a plant for monitoring the vibration of a transmission shaft. The system consists of a sensor head, i.e., an eddy current displacement sensor, and a data processing system including an amplifier, an A/D converter of 8 bits, a personal computer, and a display monitor. The Lissajous figures of the motion and the occupation distribution of the center of revolution can be obtained in real time using this system. The sampling frequency of data acquisition was $5 \mathrm{kHz}$, which is sufficiently high for the speed of $1500 \sim 1800$ cycles/min typically used in a plant. The ripple-like noise of about $30 \mu \mathrm{m}$ due to a quantization of the $\mathrm{A} / \mathrm{D}$ converter is precise enough for most plants. The system can, further, be effectively used for estimating bearing wear.

\section{References}

1) Ed. Soc. Tech. Diagnosis: A report on a technical development for a detection and a diagnosis of a light water reactor in operation-a techniques for a diagnosis of fatigue at the high working ratio, Japan, B 1/B 28 (2001)

2) Y. Sotoyama, et al : Development of a bearing wear detector for a canned motor pump, EBARA Technical Report, No. 188, Japan 34/39 (2000) 